\title{
CROSS-FLOW CAKE FILTRATION MECHANISMS AND KINETICS
}

\author{
M. R. MACKLEY and N. E. SHERMAN ${ }^{\dagger}$ \\ Department of Chemical Engineering, University of Cambridge, Cambridge CB2 3RA, U.K.
}

(First received 16 July 1991; accepted in revised form 10 January 1992)

\begin{abstract}
An experimental investigation of the cake filtration behaviour of $125-180 \mu \mathrm{m}$ polyethylene particles, suspended in a neutrally buoyant Newtonian fluid, has been carried out. Static cake filtration measurements of both filtrate volume out-flow and cake thickness kinetics show the familiar $t^{1 / 2}$ time dependence. In addition, the hydraulic resistance of the cake was found to be pressure independent, indicating that incompressible cakes were formed under the pressure conditions examined. When cross-flow is applied, the filter cake tends towards a reduced limiting thickness but surprisingly the corresponding filtration rate or flux is observed to decrease. Direct in situ observations of the cake deposition process clearly reveal the mechanisms controlling particle capture at the surface and this effect, together with a particle deposition model where the packing within the filter cake is cross-flow dependent, is used to successfully describe the observed experimental behaviour.
\end{abstract}

\section{INTRODUCTION}

Cross-flow filtration is a widely used method for the separation of phases in many types of suspension, for example water and sewage treatment (Treffry-Goatley et al., 1987), harvesting of plant and animal cells (Tanny et al., 1982), food processing (Porter and Nelson, 1972) and dewatering of mineral slurries (Hoogland et al., 1990). The phenomenon may also occur during other activities in which a suspension flows tangentially to some porous medium, e.g. fluid loss from a drilling mud resulting in the formation of a cake layer on the rock surface in a wellbore (Gray and Darley, 1980). Cross-flow has important applications in the filtration of both sub-millimetre and sub-micron particles. In the case of sub-millimetre particles, it is normal that during the filtration process a filter cake will develop and it is these kinetics, together with the associated mechanisms of the cake build-up which are the primary concerns of this paper. For sub-micron particles, the situation is more complex in that Brownian motion may play a significant role in the accumulation of particles near the filter membrane. In this case, the concept of a definite filter cake may not be appropriate and there appears to be a broad range of proposed mechanisms to explain observed effects such as concentration polarisation with Brownian back diffusion (Colton et al., 1975), or with turbulent eddy diffusivity (Hunt et al., 1987).

We choose to examine the behaviour of a slurry consisting of polyethylene particles in a neutrally bouyant Newtonian liquid. The particles are of order $100 \mu \mathrm{m}$ in size and they strongly scatter light. This makes them good for flow visualisation studies which enable us to make direct in situ observations of particle arrival and attachment at the cake surface. Using these particles, we are also able to make simultaneous

t Author to whom correspondence should be addressed. measurements of both the cake height and the filtrate flux.

\section{Background}

Previous experimental work on cross-flow filtration of sub-millimetre particles has been carried out by a number of workers. Useful examples include Porter (1972a) where the ultrafiltration of blood cells ( $\sim 5 \mu \mathrm{m})$ was examined, Hunt et al. (1987) who studied $8 \mu \mathrm{m}$ effluent (sewage) particles, Nakao et al. (1990) where observations of $1 \mu \mathrm{m}$ PMMA and $10 \mu \mathrm{m}$ $\mathrm{SiO}_{2}$ particles in water were made and Hoogland et al. (1990) who investigated $2.5 \mu \mathrm{m} \mathrm{SiO}_{2}$ slurries. Using measurements of the filtrate flux, i.e. the filtrate volume out-flow per unit area per unit time, these observations show that after an initial period of flux decline, a steady-state flux is reached. In general, this steady-state value is found to increase with increasing applied cross-flow velocity: however in a few cases, e.g. Bagdasarian et al. (1977), Fischer and Raasch (1985), Lu and Ju (1989) and Wakeman and Tarleton (1991), reduced values have also been reported which are explained in terms of the selective deposition of fine particles into the filter cake.

These experimental studies are typical in that they are limited to measurement of the filtrate flux alone because cake thickness information is not accessible. A notable exception is the work of Fordham and Ladva (1989) where the cross-flow filtration of bentonite suspensions was studied by the measurement of filtrate flux with time and post mortem measurements of the steady-state cake thickness. The steady-state flux was observed to increase with increasing cross-flow velocity, whereas the cake thickness decreased, indicating that higher shear rates reduce the cake height, and thus lowered the resistance to fluid out-flow.

Many theoretical studies have been made, leading to the development of several predictive models. 
Colton et al. (1975) modelled filtrate flux in cross-flow microfiltration using the concept of concentration polarisation. In this approach, a boundary layer containing a higher particle concentration than the bulk suspension is assumed to form adjacent to the filter medium, hence providing an additional hydraulic resistance to out-flow. To calculate a filtrate flux under steady-state conditions, the boundary layer is assumed to be in dynamic equilibrium such that the convection rate of particles towards the filter is balanced by Brownian back diffusion into the bulk suspension. The resulting model was successfully applied by Colton to the ultrafiltration of macromolecules. However, in earlier work, Porter (1972b) obtained experimental permeate fluxes for particulate suspensions that were an order of magnitude higher than those predicted by concentration polarisation.

In order to more accurately predict the limiting flux in filtration of particulate suspensions, a number of mechanisms other that Brownian back diffusion have been proposed. Some authors have extended the concentration polarisation concept: for example, Zydney and Colton (1986) incorporated an augmentation of the diffusive motion of large particles in a shear flow (i.e. shear-induced hydrodynamic diffusivity) using empirical correlations for suspensions of rigid spheres from the data of Eckstein et al. (1977). A filter length dependence of flux was predicted under steady-state conditions and results were obtained which showed good agreement with length-averaged data for blood and bacteria suspensions. In a similar approach, Hunt et al. (1987) considered the use of a turbulent eddy approach to calculate a diffusion coefficient. Good agreement with data for the filtration of aqueous calcium carbonate suspensions was obtained, provided that two empirical constants, relating diffusivity and boundary-layer thickness to the friction velocity, could be established.

Pearson and Sherwood (1988) extended the concentration polarisation model by including an analysis of a static compressible filter cake originally developed by Philip and Smiles (1982). This combination enabled cross-flow filtration to be modelled, including the growth of a cake and was applied to compressible bentonite mud. Solutions for the growth of a uniform cake are in agreement with the data obtained at early times (or quasi-static regime) by Fordham and Ladva (1989) but in the dynamic regime, steady-state solutions of the model require a scaling of length integrated filtrate flux with axial filter length, $L$, as $L^{2 / 3}$ whereas the data demonstrate linearity in $L$.

Other concepts have been considered, including erosion by turbulent bursts (Gutman, 1977) particle migration due to inertial lift (Green and Belfort, 1980) scour removal (Fane, 1984) and shear-enhanced hydrodynamic diffusion (Romero and Davis, 1988, 1990; Davis and Sherwood, 1990). These models, together with concentration polarisation, can be summarised as being those which employ the concept of a backdiffusion mechanism in order to achieve steady-state conditions and, for those which are mathematically rigorous, a length-dependent flux is predicted. Another genre exists that may be described as particle adhesion models. Examples in this field include the critical flow model of Rautenbach and Schock (1988) which assumes that cake deposition ceases when the feed velocity exceeds some multiple of the filtrate flux, the constant of proportionality being determined empirically for a given suspension. Similarly, Blake et al. (1990) equated the ratio of net tangential to net normal forces with a coefficient of friction at the cake surface. From this analysis, using the critical point at which the tangential force just exceeds frictional drag, an expression for steady-state flux can be obtained in terms of surface shear rate, particle radius and two constants for a given particulate system. This was shown to be applicable for suspensions of 0.5 and $1.8 \mu \mathrm{m}$ radius polystyrene spheres. Finally, a numerical simulation of a particle adhesion style model has recently been reported by Schmitz et al. (1990).

\section{EXPERIMENTAL}

In order to identify the relevant physical mechanisms for dynamic filtration of a given system, direct optical observations of the process are required and a well-characterised suspension in which the particles can be individually resolved by a video camera is necessary. In view of this, a model suspension consisting of neutrally buoyant polyethylene particles dispersed in a water/methylated spirit mixture at a solids volume concentration of approximately $0.05 \%$ was used. The polyethylene particles were aquaphobic, so to produce stable suspensions they were soaked in detergent prior to being dispersed. Hence, some detergent was present in the liquid phase, the viscosity of which was found to be $\eta=2.38 \mathrm{mPa} \mathrm{s}$. The particles used were in the size range $125-180 \mu \mathrm{m}$, and this relatively narrow distribution was chosen in order that the effects of the deposition of fine particles into the filter cake structure could be avoided. The filtration properties of the model suspension were then investigated under both static and dynamic conditions.

\section{Static filtration}

The apparatus for static filtration experiments where the cumulative filtrate mass and filter cake height were simultaneously measured, is shown schematically in Fig. 1. A stainless-steel filter mesh was chosen to facilitate operation at low pressure drops (maximum $\sim 10 \mathrm{kPa}$ ) as the open structure provided negligible additional hydraulic resistance to that of filter cake formed by the polyethylene particles. In order to start an experimental run, a bung was removed from the base of the column and the filtrate mass data collection routine of the microcomputer was initialised by a keyboard command. Monitoring of the filter cake growth was achieved using a recording made with the $15 x$-magnification video camera. This was played back later and cake height data as a function of time was obtained by 
taking measurements directly from the video screen and scaling by the magnification. The camera was set up to begin recording before the bung was removed, so that the start of the experiment could be clearly seen on the play-back. Thus, the two sets of data could

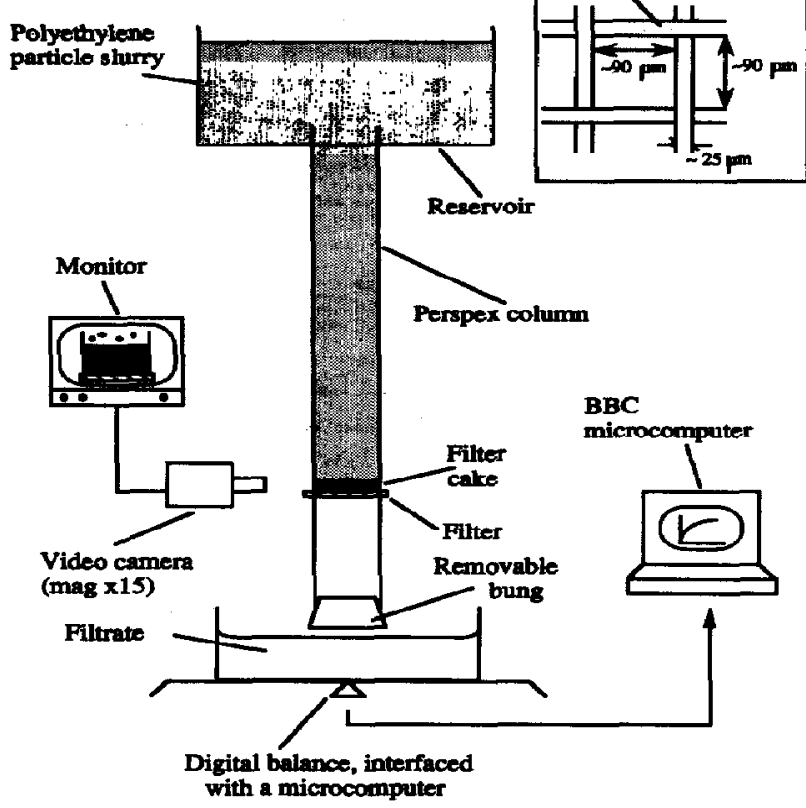

Fig. 1. Schematic diagram of static filtration apparatus. be synchronised, and hence simultaneous measurements of the cumulative filtrate mass and the filter cake thickness were obtained: These measurements were made for a range of constant filtration pressure $(\Delta P)$, i.e. about 2 to $10 \mathrm{kPa}$ and, in each experiment, the' reservoir level was maintained to give the required constant $\Delta P$-value.

\section{Cross-flow filtration}

Filtration experiments under cross-flow conditions were performed using the apparatus shown schematically in Fig. 2. The Perspex test section was $1 \mathrm{~m}$ long, with the width $(30 \mathrm{~mm})$ and height $(10 \mathrm{~mm})$ chosen to give an essentially $2 D$ channel flow. The first $700 \mathrm{~mm}$ of length allowed for flow development prior to arrival at the filter which was $100 \mathrm{~mm}$ long in the direction of cross-flow, by $7 \mathrm{~mm}$ wide. This part of the test section was illuminated with a planar beam that was a few particle diameters wide, thus reducing the number of particles in view at any time and providing clarity of observation through the glass window.

In each experiment, a constant inlet net cross-flow velocity $(v)$ and filtration pressure $(\Delta P)$ were set up and maintained by adjustment of the flow control valves. $\bar{v}$ and $\Delta P$ were monitored using a turbine flow meter and a pressure transducer, respectively. Filtration was initiated using the microcomputer to synchronise opening of the solenoid valve with the start of data collection from the balance, which measured the cumulative filtrate mass as a function of time. On completion of the experimental run, these measurements were converted into filtrate flux data, by differentiating with respect to time. The cake height was continuously monitored during the filtration process

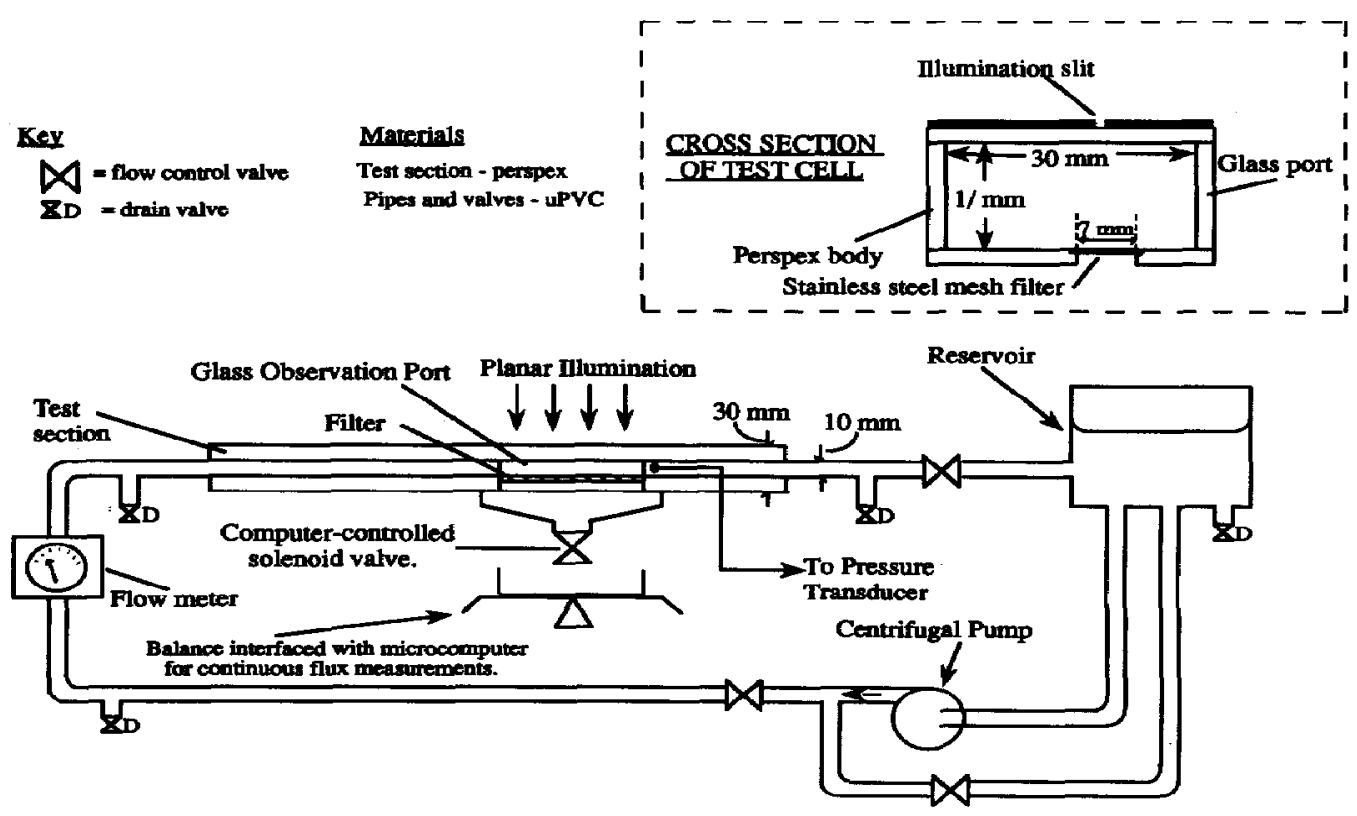

Fig. 2. Schematic diagram of cross-flow filtration apparatus. 
using the video camera in the same manner as described for the static filtration experiments. This procedure was repeated for a $\bar{v}$ range of about 0 to $0.25 \mathrm{~m} / \mathrm{s}$ at a constant $\Delta P$ of $5 \mathrm{kPa}$, being in the midrange of the $\Delta P$-values examined in static filtration and also much greater than the pressure drop along the filter channel (i.e. $\sim 0.1 \mathrm{kPa}$ ).

Further, with $\bar{v}$ and $\Delta P$ maintained at $0.1 \mathrm{~m} / \mathrm{s}$ and $5 \mathrm{kPa}$, respectively, the cumulative filtrate out-flow was measured for a series of filter lengths in the range $1-10 \mathrm{~cm}$. This was achieved using rubber bungs in the test section base to 'blind' successively larger lengths of the filter.

Optical observations of individual particle behaviour at the filter cake-slurry interface, during cake growth, were made by examining the video recordings in slow-motion replay from which still frames at $1 / 50 \mathrm{~s}$ intervals could be obtained. Higher quality stationary images of the global behaviour during the filtration process were obtained using a $35 \mathrm{~mm}$ SLR camera.

\section{RESULTS}

\section{Static filtration kinetics}

Figures 3 and 4 show filter cake height $(h)$ and cumulative filtrate mass, respectively, plotted as a function of the square root of time $\left(t^{1 / 2}\right)$ for a range of $\Delta P$. Within the bounds of experimental accuracy, i.e. about $\pm 0.3 \mathrm{~mm}$ in cake height and $\pm 1 \mathrm{~g}$ in filtrate mass, both parameters appear to increase linearly with $t^{1 / 2}$ over the chosen pressure range, provided that, in the case of the filtrate mass, non-zero time axis intercepts are accepted. These intercepts are not surprising as the filtration experiment and mass data collection were started by hand leading to synchronisation errors of about \pm 1 s. Further, as the reservoir was refilled manually, maintainance of the static head in the initial rapid fluid loss period (in this case $\sim 2 \mathrm{~s}$ ) was difficult and shortfalls of the order $0.2 \mathrm{kPa}$ occurred. Thus, some early time curvature would be introduced to the mass data as $\Delta P$ rose to the required value.

The $t^{1 / 2}$ kinetics for cake height growth and cumulative filtrate mass are consistent with predictions from well-known static filtration theory [see, e.g. Akers and Ward (1977)] using Darcy's law for flow through a packed bed and a simple mass balance on the solid phase. Assuming that the cake formed is incompressible and of constant void volume fraction (E) and that no particles are left in the filtrate, the cake height $h$, is given simply by

$$
h=\left(\frac{2 \Delta P s t}{R(1-\varepsilon-s)}\right)^{1 / 2}
$$

where $s$ is the particle volume fraction in the suspension and $R$ is the specific resistance of the cake which can be defined from Darcy's law, i.e.

$$
\boldsymbol{R}=\frac{\Delta P}{h u}
$$

where $u$ is the filtrate flux. This analysis predicts that the cake height grows linearly with $t^{1 / 2}$ and similarly, $t^{1 / 2}$ kinetics are found for the cumulative filtrate mass, $M$, as

$$
M=\rho A \int_{0}^{t} u \mathrm{~d} t=\rho A\left(\frac{2 \Delta P(1-\varepsilon-s)}{R s} t\right)^{1 / 2}
$$

Cake Height vs. Time $1 / 2$

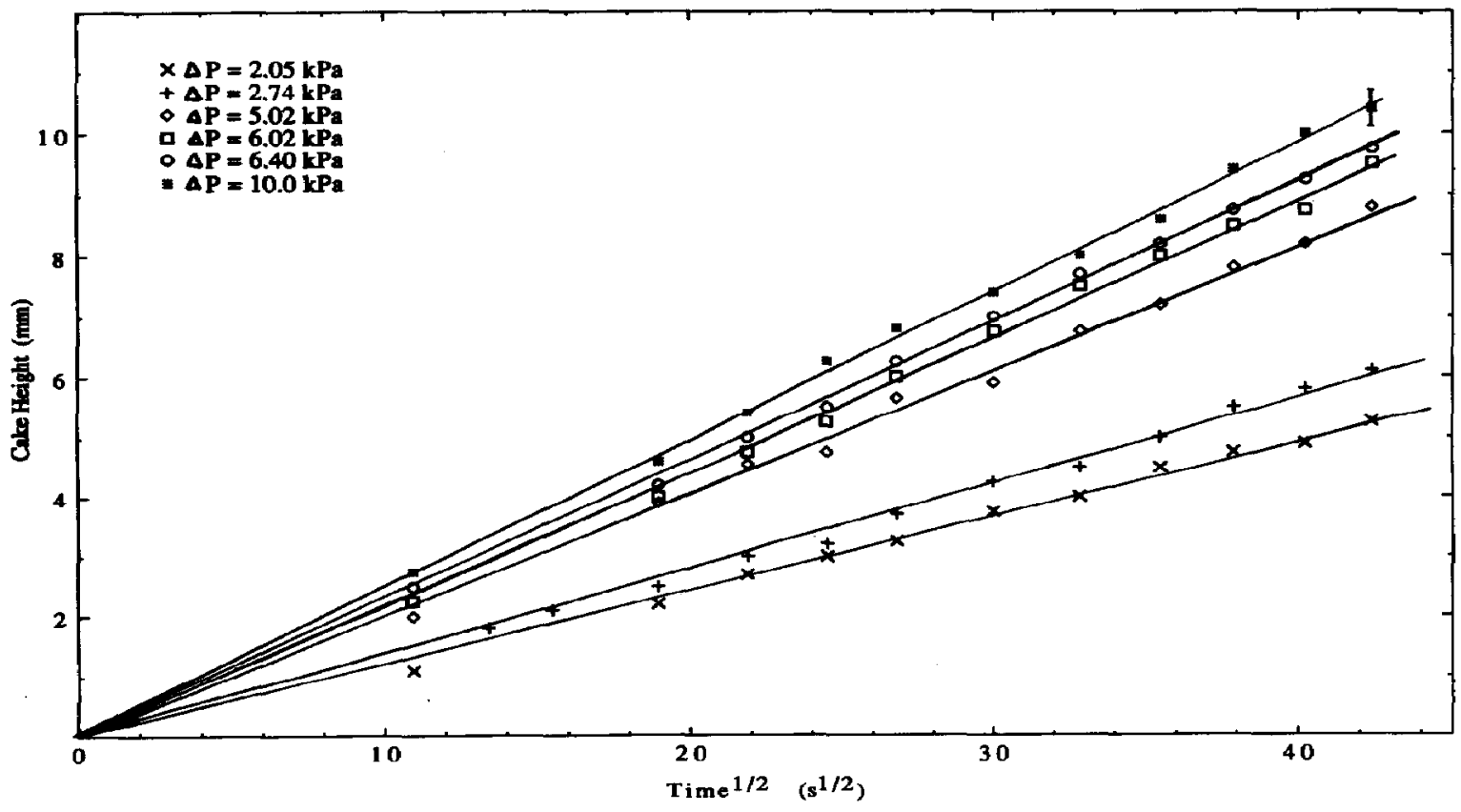

Fig. 3. Cake height vs time $e^{1 / 2}$. Static filtration data. 


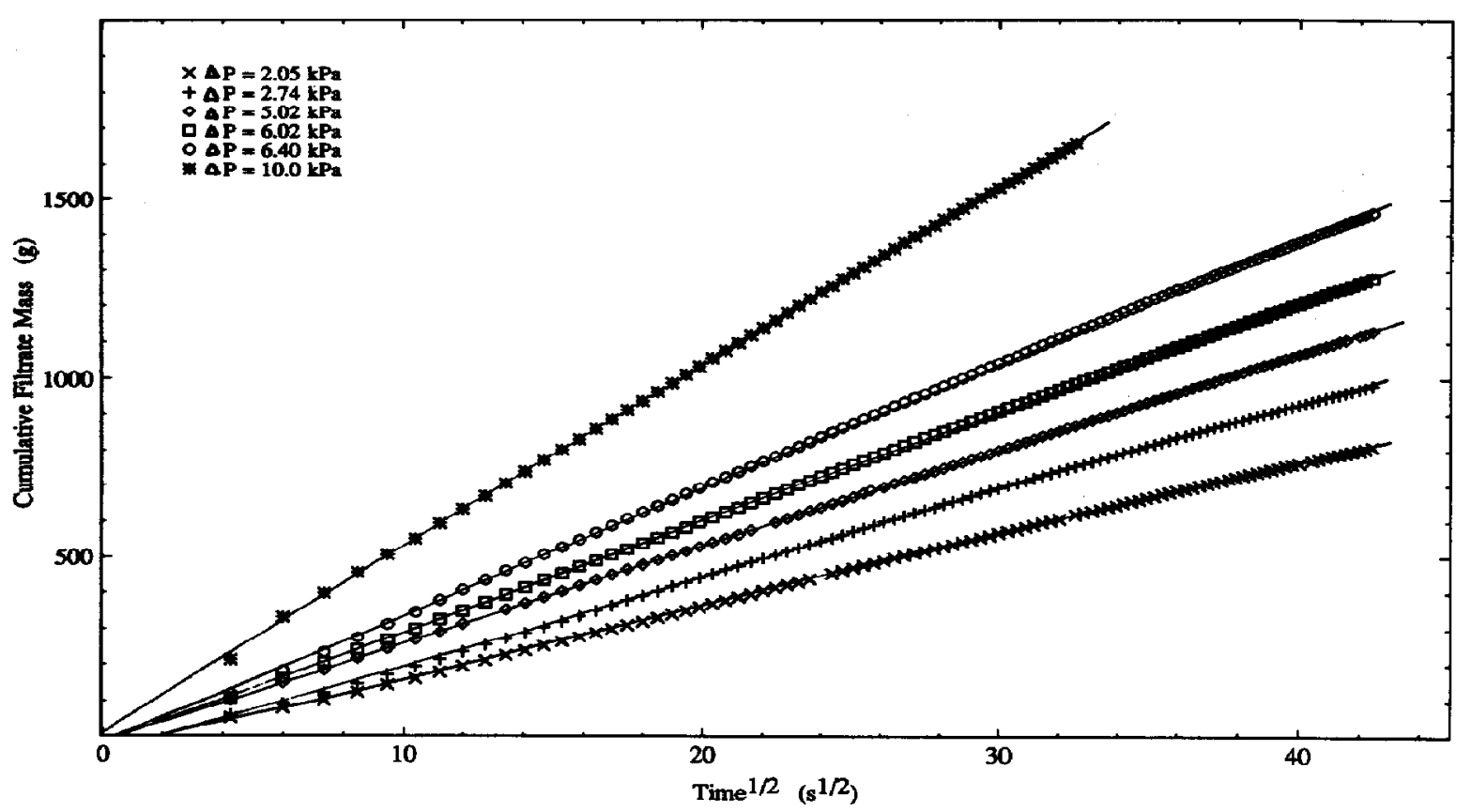

Fig. 4. Cumulative filtrate mass vs time $e^{1 / 2}$. Static filtration data.

Table 1. $10^{-6} R_{\left(\mathrm{N} \mathrm{s} \mathrm{m}^{-4}\right)}$

\begin{tabular}{|c|c|c|c|c|c|c|c|}
\hline \multirow[b]{2}{*}{ Time (min) } & \multicolumn{7}{|c|}{$\Delta P(\mathrm{kPa})$} \\
\hline & 2.05 & 2.74 & 4.56 & 5.02 & 6.02 & 6.40 & 10.0 \\
\hline 6 & 176 & 194 & 189 & 182 & 188 & 176 & 171 \\
\hline 8 & 166 & 169 & 195 & 179 & 186 & 167 & 173 \\
\hline 10 & 170 & 177 & 180 & 184 & 189 & 173 & 167 \\
\hline 12 & 178 & 186 & 181 & 185 & 182 & 177 & 171 \\
\hline 15 & 174 & 181 & 189 & 190 & 188 & 177 & 177 \\
\hline 18 & 179 & 180 & 190 & 193 & 190 & 181 & 173 \\
\hline 21 & 176 & 179 & 190 & 193 & 190 & 184 & 173 \\
\hline 24 & 179 & 182 & 181 & 194 & 184 & 180 & 165 \\
\hline 27 & 177 & 180 & 180 & 195 & 194 & 184 & 164 \\
\hline 30 & 180 & 183 & 195 & 191 & 189 & 184 & 170 \\
\hline Mean \pm s.d. & $175 \pm 4$ & $181 \pm 6$ & $187 \pm 5$ & $188 \pm 5$ & $187 \pm 3$ & $178 \pm 5$ & $171 \pm 4$ \\
\hline
\end{tabular}

$R=\Delta P / h u$. Experimental errors $h \sim 5 \%, u \sim 2 \%, \Delta P \sim 1 \%$, hence expect $R \sim \pm 8 \%$.

where $\rho$ is the density of the fluid phase and $A$ is the filter area. Thus, the results given in Figs 3 and 4 are consistent with the formation of incompressible cakes, over the $\Delta P$ range examined. Also, by differentiating the cumulative mass data with respect to time, values of $u$ were obtained and together with the cake height data, values of the specific cake resistance, $R$, were calculated using eq. (2). These results are given in Table 1 and they show that $R$ is constant within the experimental error, confirming that the model suspension forms incompressible filter cakes over the $\Delta P$ range $\sim 2-10 \mathrm{kPa}$.

From the slopes of the cumulative mass curves in Fig. 4, we can obtain a value for $\lambda=s /(1-\varepsilon-s)$ using eq. (3). For cxample in the case of $\Delta P=5 \mathrm{kPa}$, $\mathrm{d} M / \mathrm{d} t^{1 / 2}=(25 \pm 0.5) \mathrm{g} / \mathrm{s}^{1 / 2}$, given $A=1.13 \times 10^{-4} \mathrm{~m}^{2}$, $\rho=940 \mathrm{~kg} / \mathrm{m}^{3}$ and the mean value $R=(1.80 \pm 0.1)$ $\times 10^{8} \mathrm{Ns} \mathrm{m}^{-4}$, we obtain $\lambda=(1 \pm 0.1) \times 10^{-3}$. Similarly, from the corresponding cake height data in Fig. 3 and using eq. (1), we calculate $\lambda=(8 \pm 0.8)$ $\times 10^{-4}$, showing fair agreement in view of the experimental precision in the cake height data. From $\lambda$, we can estimate the cake porosity, $\varepsilon$, if $s$ is known. The exact value of the slurry concentration at the filter is somewhat difficult to accurately access. The starting concentration was $s=0.05 \%$ but the particles are aquaphobic and the detergent soaking procedure is not $100 \%$ efficient. This means that some particle 
flocculation occurred within the flow loop of the apparatus in which the slurry was mixed (shown in Fig. 2) leading to precipitation in the holding tank. The slurry used for the static filtration was taken from this apparatus. By filtering two $250 \mathrm{ml}$ samples taken from the fluid stream and drying then weighing the solids, a volume concentration of approximately $0.03 \%$ was measured. In view of the very low solids volume fraction, this method is very sensitive to loss of solid mass and local fluctuations in concentration, and therefore our precision in $s$ is not good enough to accurately predict $\varepsilon$. However, $s$ must lie between an upper and lower bound of $s=0.05 \%$ and $s=0.03 \%$. Using the two values of $\lambda$ that have been determined from the cumulative mass and cake height data, we have an upper and lower bound value of the cake voidage given by 0.70 and 0.37 which is plausible when compared for example with random loose packing where $\varepsilon \sim 0.44$.

\section{Cross-flow filtration}

Kinetics. The results obtained for cake height, $h$, and filtrate flux as a function of time are shown in Figs 5 and 6, respectively. The measurements were all made at $\Delta P=5 \mathrm{kPa}$ for a mean channel cross-flow velocity $(\vec{v})$ range of 0 to $0.24 \mathrm{~m} / \mathrm{s}$. This corresponds to the laminar flow regime given by defining the Reynolds number as $R e=(\bar{v} H \rho) / \eta$, where $H$ is the channel height, i.e. $10 \mathrm{~mm}$, and for the fluid $\rho=940 \mathrm{~kg} / \mathrm{m}^{3}$ and $\eta=2.38 \mathrm{mPa} \mathrm{s}$; then for the highest flow rate $R e_{\max } \sim 950$. Further, for a particle rolling at the wall, the effective shear rate is given approximately by $\gamma_{w} \sim v / d$, where $d$ is the particle diameter and $v$ is the fluid velocity one particle diameter away from the wall. Assuming a mean diameter of $\sim 150 \mu \mathrm{m}$ and a parabolic velocity profile in the channel, $v \sim 0.1 \vec{v}$, and therefore $\gamma_{w(\max )} \sim 160 \mathrm{~s}^{-1}$.

From Fig. 5 it can be seen that the cross-fiow produces cakes that approach limiting thickness which decreases with increasing $\bar{v}$. Surprisingly however, in Fig. 6 the corresponding filtrate flux decreases as the cross-flow velocity increases. This indicates that thinner cakes produced at higher cross-flow velocities offer more hydraulic resistance than thicker ones formed at lower velocities. The operating pressure conditions have been chosen to be in the range where the particles have been shown to form incompressible cakes. Therefore, this effect cannot be explained by the subsequent compaction of existing cake.

In order to explore this reduced flux effect further, a filter cake was grown under high cross-flow $(\bar{v} \sim 0.24 \mathrm{~m} / \mathrm{s})$ and constant low flux $(u \sim 0.01 \mathrm{~m} / \mathrm{s})$ conditions in order that a cake could be grown where the angle of approach of particles was very narrow at all times. Then the fluid out-flow through the cake was measured with no cross-flow applied. The specific cake resistance was then calculated, in the manner described earlier, and compared with the value found from the static filtration experiments, i.e.

(a) statically grown cake

$$
R_{1} \sim(1.8 \pm 0.1) \times 10^{8} \mathrm{Ns} \mathrm{m}^{-4}
$$

(b) cake grown in cross-flow

$$
R_{2} \sim(5.5 \pm 0.4) \times 10^{8} \mathrm{Ns} \mathrm{m}^{-4}
$$

and so the ratio $R_{2} / R_{1} \sim(3 \pm 0.5)$ confirming that the

Cake Thickness vs. Time.

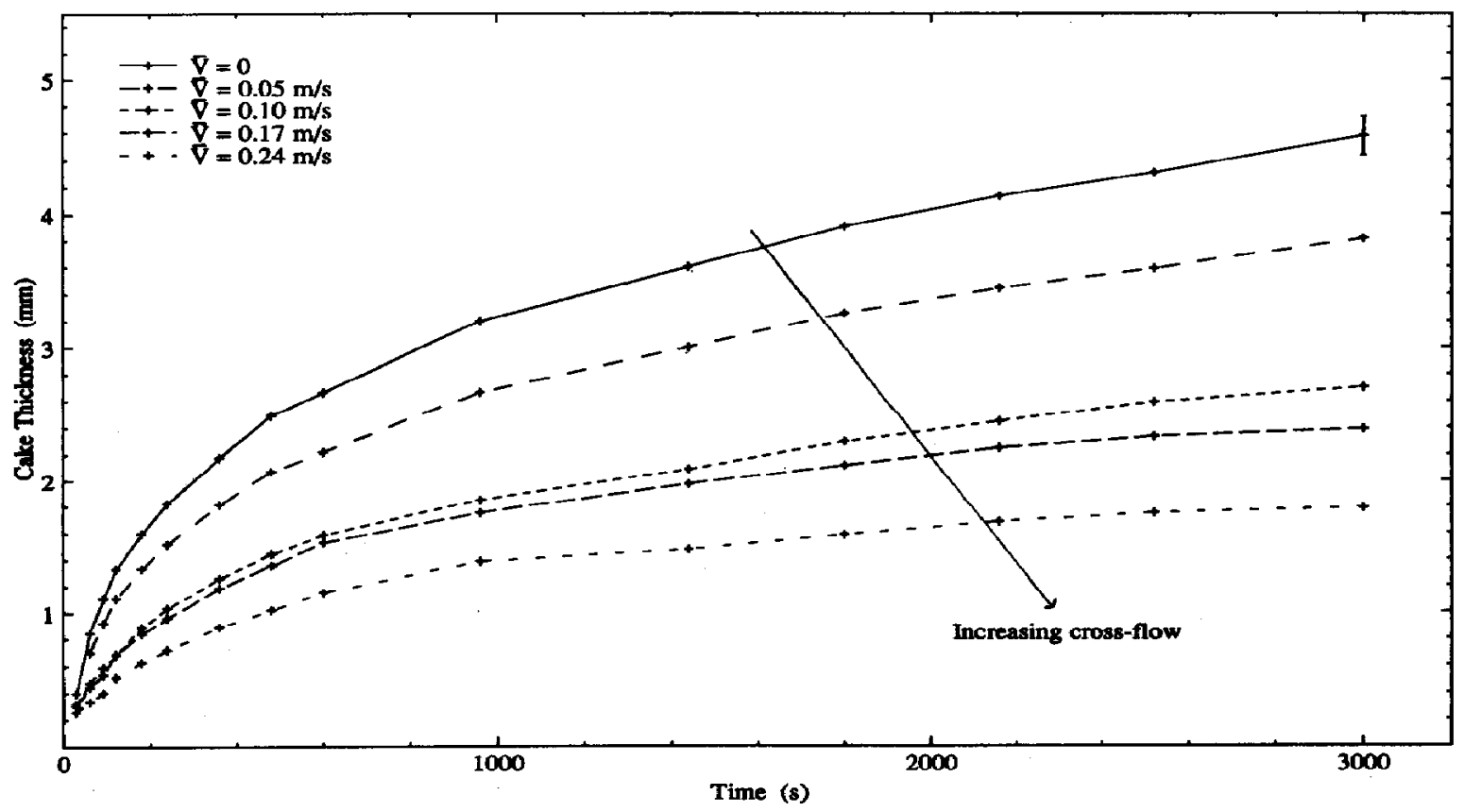

Fig. 5. Cake thickness vs time. Cross-flow filtration deta. 
Filtrate Flux vs. Time.

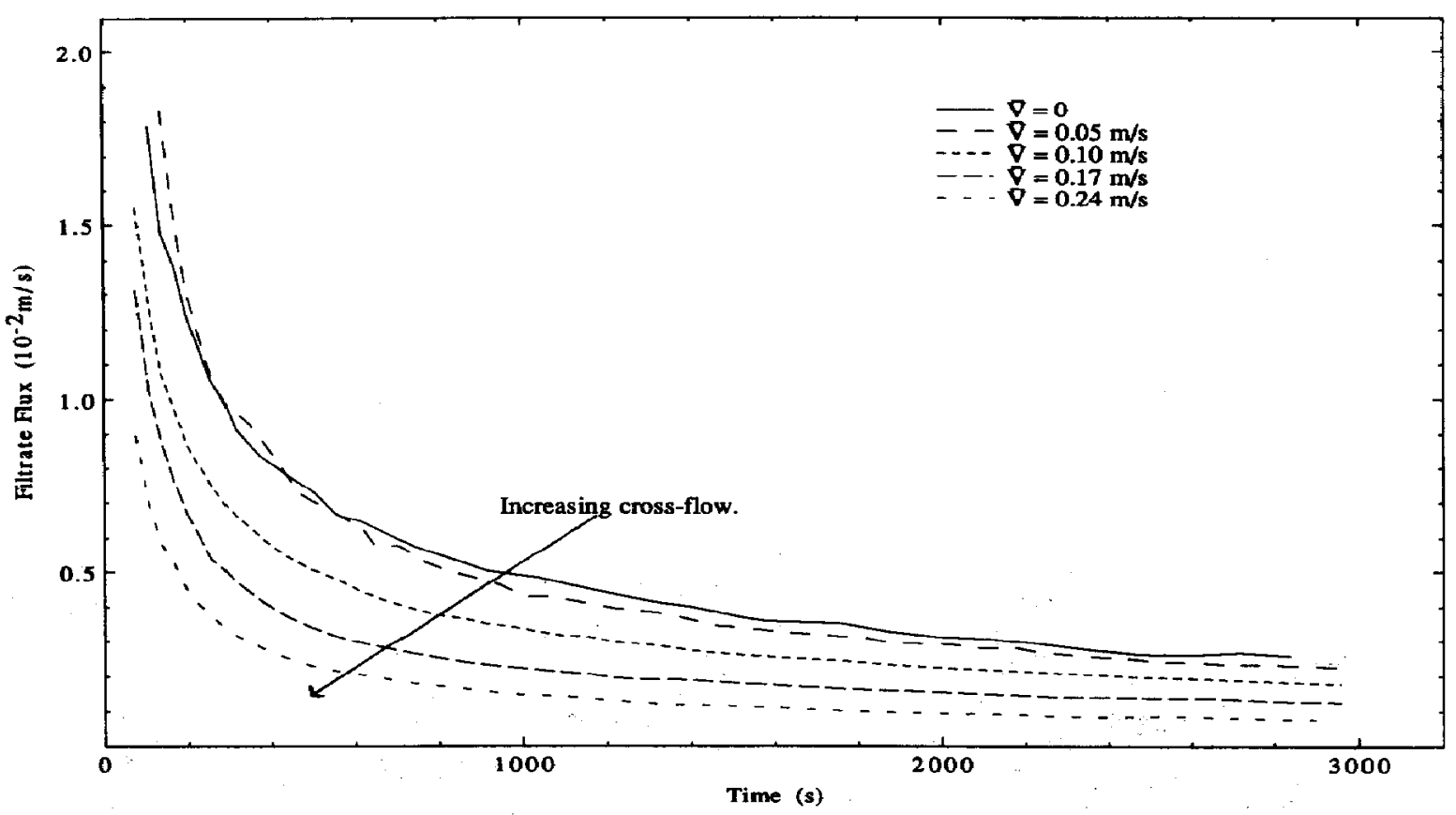

Fig. 6. Filtrate flux vs time. Cross-flow filtration data.

imposition of cross-flow produces cakes of higher specific resistance than those found in static filtration.

In a similar way to the static filtration experiments, the data obtained in the cross-flow apparatus for $\bar{\theta}=0$ can be used to determine $\lambda$ using eqs (1) and (2). Given that $\Delta P=5 \mathrm{kPa}$ and $R \sim(1.8 \pm 0.1)$ $\times 10^{8} \mathrm{~N} \mathrm{~s} \mathrm{~m}^{-4}$ we find $\lambda=(5.6 \pm 0.3) \times 10^{-4}$, which is in poor agreement with the static filtration value. However this is not surprising because, as shown in Fig. 2, the filter mesh is not bounded at the sides, and therefore the cake build up is strictly $3 D$, including side and end effects which we would expect to modify the kinetics. Nevertheless taking $s \sim 0.03 \%$, we obtain an effective $\varepsilon \sim 0.46$ which is credible; cf. random loose packing, i.e. $\varepsilon \sim 0.44$.

Optical observations. The optical observations are summarised by Figs 7(a)-(d) and 8(a) and (b), in which the dotted line marks the filter position and $H$ is the channel height of $10 \mathrm{~mm}$. This set of photographs was obtained at a low cross-flow velocity $(\bar{v} \sim 0.025 \mathrm{~m} / \mathrm{s})$ which was necessary for clear flow visualisation using the $35 \mathrm{~mm}$ camera. Although the timescale of these results is different to those shown in Fig. 6, the mechanisms shown are qualitatively identical to those observed for all the cross-flow conditions studied. In Fig. 7 (a) the early stage of cross-flow filtration is shown. In this case, the filtrate flux dominates the fluid mechanics and particles are advected directly onto the filter cake. The particles remain in the position where initial impact occurs. As the cake thickness increases the filtrate flux at constant differential pressure decreases. Figure 7(b) shows the situation where the cross-flow velocities are comparable to the filtrate velocities and the particles take a parabolic form of trajectory. In this situation also, the particles generally remain where the initial impact with the interface occurs.

At later stages in the filtration the situation changes. As the cake thickness increases, the crossflow velocity dominates the fluid mechanics. In this case, shown in Fig. 7(c) and (d), flow trajectories are nearly parallel to the cake. Particles that arrive at the rough cake surface shown in Fig. 7(c) are seen to roll until they find an edge or crevice at which to become captured onto the cake. The initial point of impact is invariably not the final position of capture. This effect is shown by a video sequence of time interval $1 / 25 \mathrm{~s}$, given in Fig. 8(a) and (b). In the case of Fig. 8(a), a particle is tracked moving along the surface and then finding a permanent residence. site within the field of view of the camera. In Fig. 8(b), taken at a later stage of cross-flow, a particle is seen to roll along the generally smooth surface and not be captured at all.

From these observations we gain the clear impression that capture of particles at the surface is controlled by the relative magnitude of filtrate velocity and cross-flow velocity near the interface. In addition, we gain the impression that particle packing will also be influenced by this relative velocity. When the filtrate flux dominates, packing is non-selective and random. When cross-flow dominates, the particles are highly selective as to where they finally reside and packing efficiency will be increased. We did not see any evidence in this fluid suspension for back diffusion or flux of particles away from the cake surface. 
Filter length dependence. The sections of cake shown in Figs 7 and 8 appear to be of uniform height and this was observed to be the case over the whole filter length of $100 \mathrm{~mm}$, apart from small ( $\sim 5 \mathrm{~mm})$ regions at the entry and exit. Measurements of the dynamic filtration rate $(\mathrm{d} M / \mathrm{d} t)$ at constant $\Delta P$ and $\bar{v}$ were made for filters of different length. The data are shown in Fig. 9 and were obtained in the dynamic regime after a filtration time of $1800 \mathrm{~s}$. The size of the data symbols reflects the measurements accuracy and it can be seen that a good fit is obtained by a straight line with an RMS error of $5 \%$ provided that lengths less than the observed development regions are ignored. This is consistent with the existence of con- stant local filtrate flux along the filter length, together with the effects of the end regions and fluid flow through the sides of the cake which increase the effective filtration area. It is reasonable to expect the side effects to vary proportional to $L$, but the end effects would mainly affect the data at small filter lengths and may lead to a disproportionate change in filtration rate with filter length in this region.

Some cross-flow filtration theories, such as those based on concentration polarisation, require the local filtrate flux, $u(l)$, to decrease along the filter length as $l^{1 / 3}$ in order to predict steady-state (or dynamicregime) conditions, i.e. $u(l)$ proportional to $l^{-1 / 3}$. Thus, the length integrated flux would be propor-
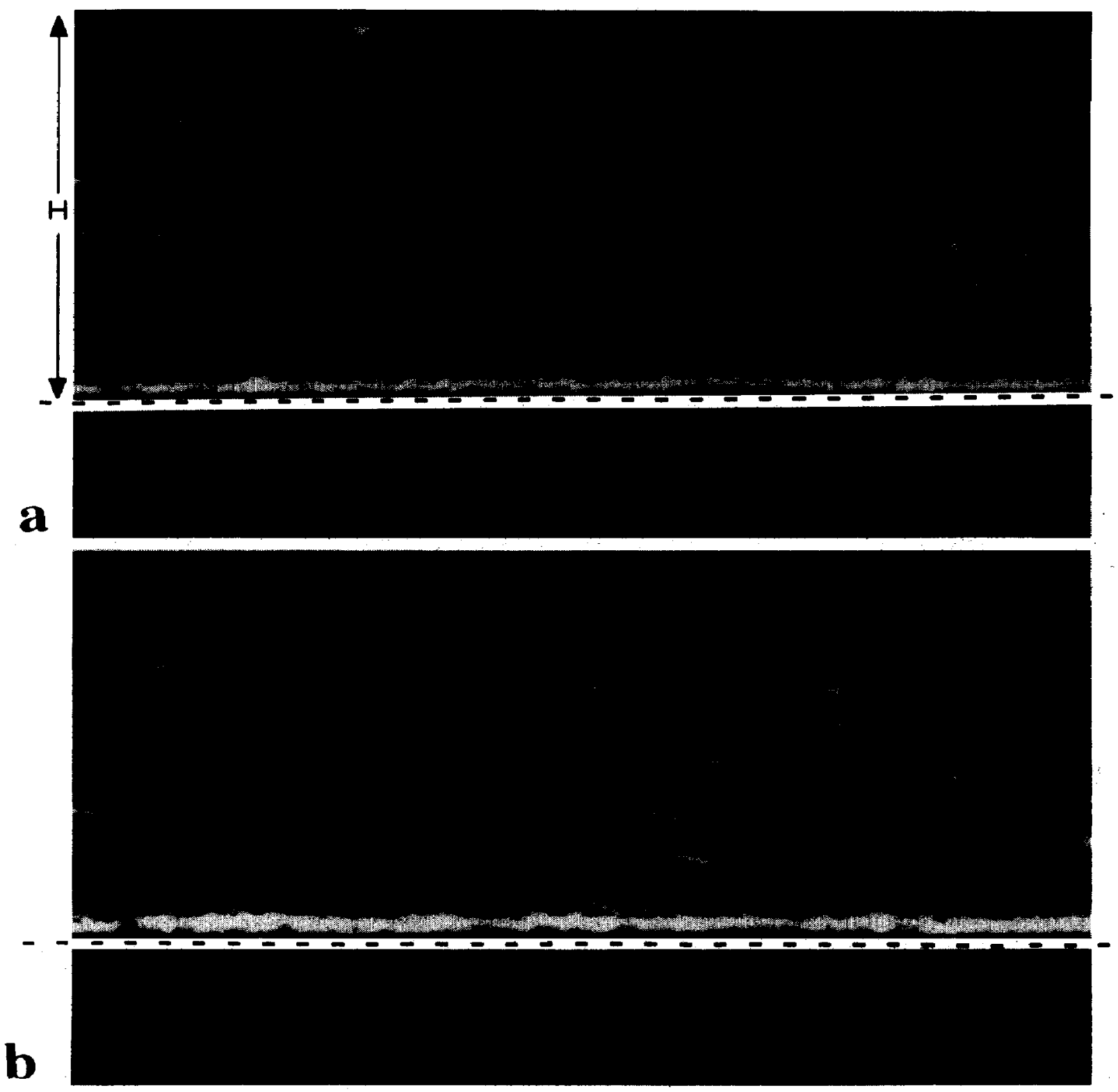

Fig. 7(a)-(b). 

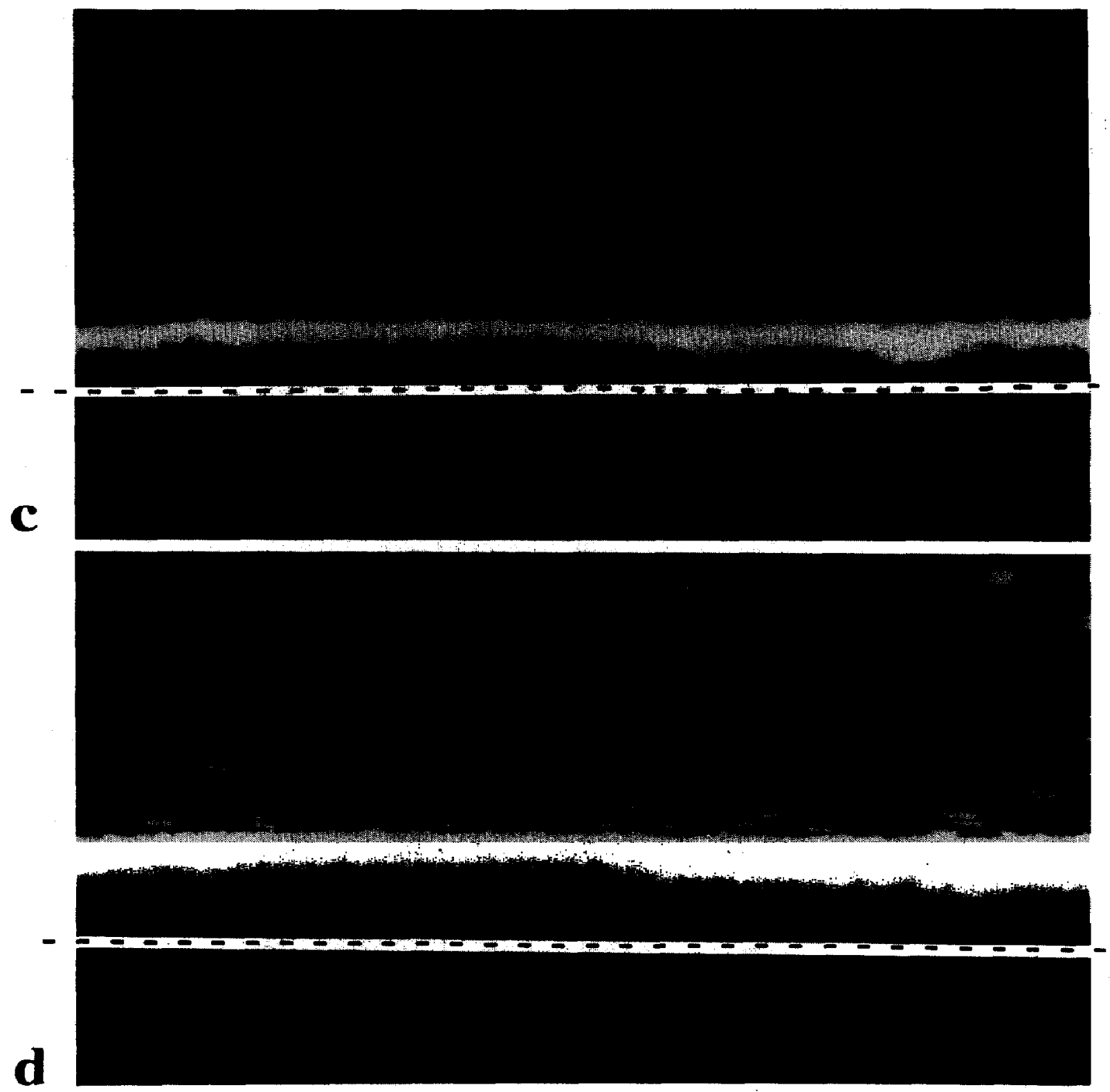

Fig. 7. Flow visualisation photographs of the filtration process. (a) Early time behaviour $t \sim 3 \mathrm{~s}$, (b) and (c) intermediate behaviour $t \sim 3$ and $10 \mathrm{~min}$, respectively, (d) limiting behaviour $t \sim 20 \mathrm{~min}$. $H$ is the channel height of $1 \mathrm{~cm}$. The dotted line represents the filter. $\bar{v}=0.025 \mathrm{~m} / \mathrm{s}, \Delta P \sim 5 \mathrm{kPa}$.

tional to $L^{2 / 3}$. If this was correct the data in Fig. 9 should be linearly related to $L^{2 / 3}$ rather that $L$. In order to investigate this, a least-squares routine was used to fit the filtration rate data to the form $k_{1} L^{n}$ $+k_{2}$, where $k_{2}$ represents the end effects. The best fit with RMS error $\sim 4.5 \%$ was obtained for $n=0.91$, which is closer to a linear relationship than to $L^{2 / 3}$. This is consistent with the equivalent results of Fordham and Ladva (1989) obtained for bentonite muds, where a linear relationship was found to provide the best fit. It is worth noting that if the end effects are neglected and the data in Fig. 9 is fitted to $k_{1} L^{2 / 3}$ an acceptable fit of RMS error $\sim 6 \%$ can be obtained. It is therefore difficult to draw any precise general conclusions on the issue of the length dependence of filtrate flux. However, in view of the good linear fit obtained and the uniform cake heights observed, it appears that for our system the filtrate flux is length independent provided that $L$ is large compared with the scale of the entry and exit regions of the cake.

\section{MODELLING}

It has been shown that for our particulate suspension, the imposition of cross-flow produced filter 
cakes of higher hydrodynamic resistance than those formed under static conditions Also the consequent dynamic filtrate flux was found to be length independent and in our optical observations, no evidence of any particle back-diffusion mechanism was seen. In view of these points, it would appear that there is no theoretical model in the literature which is fully appropriate to our system. Hence, we develop a model

9

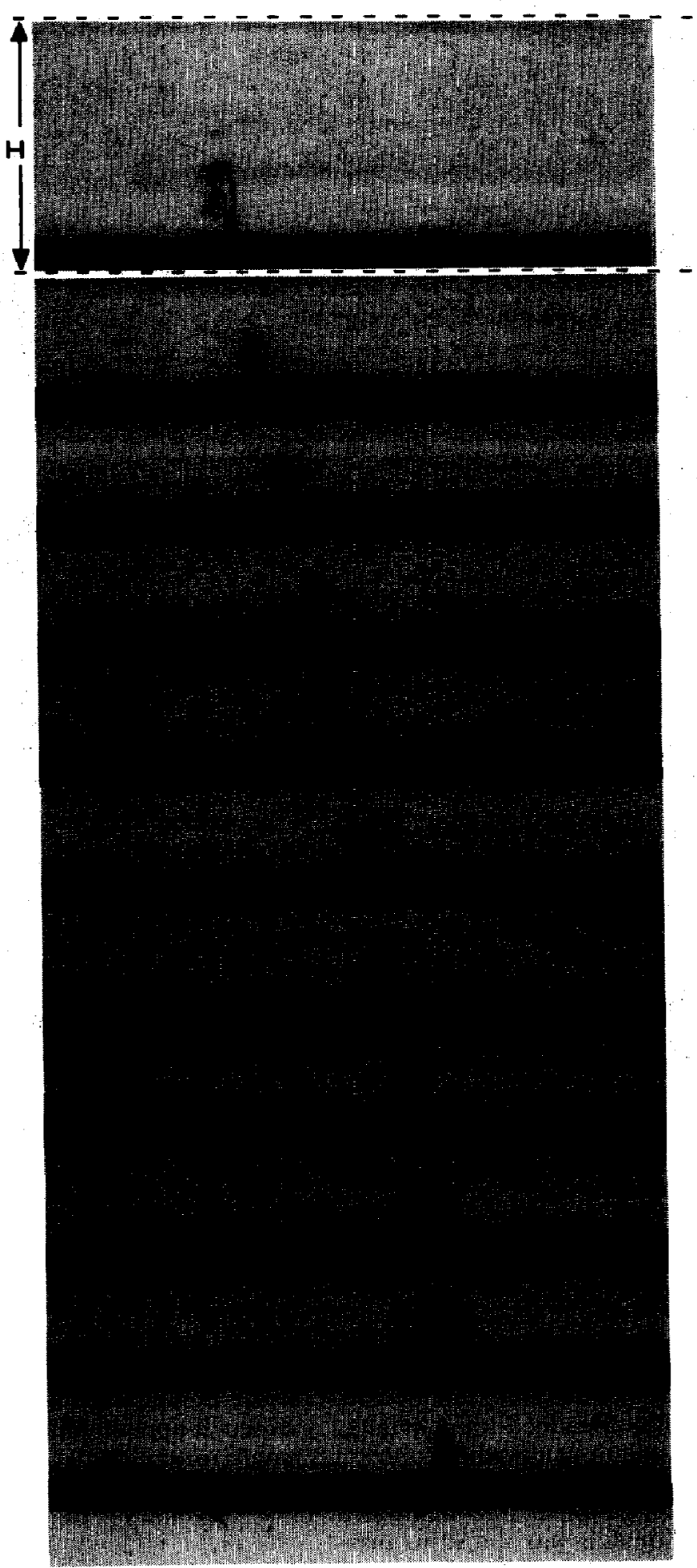

Fig. 8(a). 
b

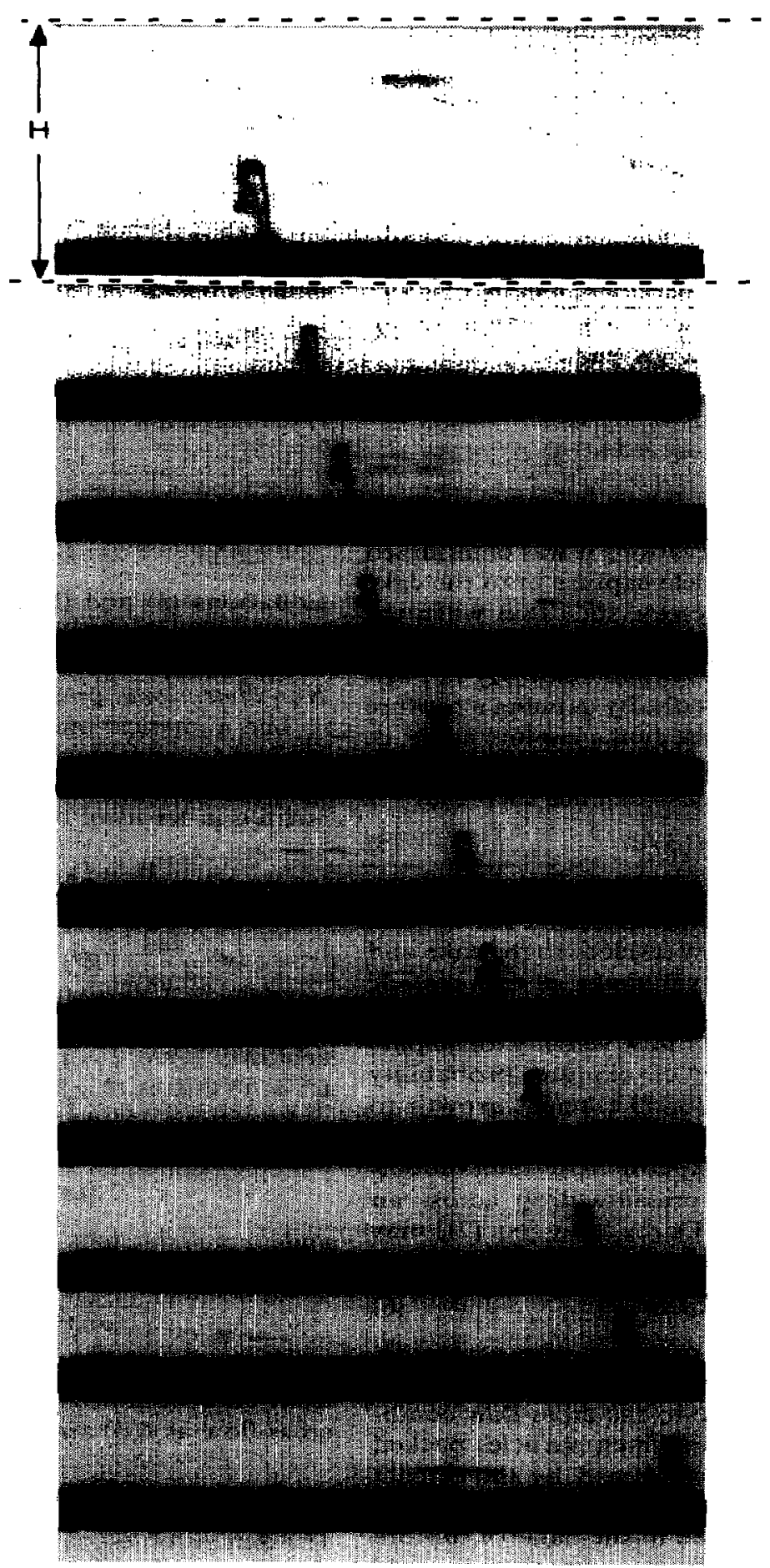

Fig. 8. Video still frames of limiting behaviour. (a) Particle residence site selection, (b) particle rolling and exit. Time interval between frames is $1 / 25 \mathrm{~s}$. $H$ is the channel height of $1 \mathrm{~cm}$. The dotted line represents the filter. Finger highlights position of particle. $\bar{v} \sim 0.025 \mathrm{~m} / \mathrm{s}, \Delta P=5 \mathrm{kPa}$.

below, which is consistent with both our local observations and global measurements.

In static filtration the rate of cake height growth can be predicted from a simple mass balance on the solid phase, assuming that the cake formed is incom- pressible and of constant void fraction $(\varepsilon)$ and that no particles are left in the filtrate, then

$$
\frac{\mathrm{d} h}{\mathrm{~d} t}=u \frac{s}{(1-\varepsilon-s)}
$$


Filtration Rate vs. Filter Length

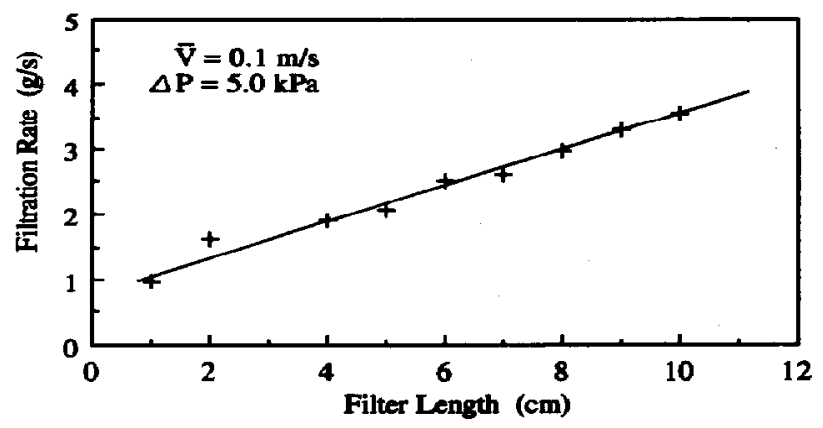

Fig. 9. Filtration rate vs filter length. Dynamic regime data.

where $s$ is the solid volume fraction and $u$ is the filtrate flux. From the optical observations it was determined that the probability of particle capture onto the cake was related to arrival trajectory, such that when the filtrate flux dominates all impacting particles are captured and as cross-flow becomes increasingly important the capture probability falls. By defining a capture probability function $f(\phi)$, based on a capture angle, $\phi$, as defined in Fig. 10, we can write a modified expression for the filtration mass balance such that

$$
\mathrm{d} h=\lambda u f(\phi) \mathrm{d} t
$$

where $\lambda=s /(1-\varepsilon-s)$. The angle $\phi$, and bence $f(\phi)$, is based on the relative magnitudes of the filtrate flux $(u)$ and the cross-flow velocity $(v)$ close to the cake and we have chosen to use the value of $v$ at one particle diameter from the surface as being a realistic representation of the effective cross-flow velocity for impacting particles. The choice of capture probability function will therefore relate cake height growth to impact trajectory, and so $f(\phi)$ must be determined, qualitatively at least, from experimental observations.

In order to predict the pressure drop across an incompressible packed-bed Darcy's law [eq. (2)] may be used, which in differential form becomes

$$
\mathrm{dP}=\boldsymbol{R} \boldsymbol{d} \mathrm{h}
$$

where $\mathrm{d} P$ is the elemental pressure drop across $\mathrm{d} h$. From our experimental results it was found that the cake resistance was sensitive to the cross-flow conditions and that the manner in which particles packed on the cake surface was determined by the impact trajectory. Thus, assuming that the resistance of an elemental cake layer is related to the angle of capture and taking into account that our experiments were conducted at constant differential pressure, we may write

$$
\Delta P=\int_{h_{0}}^{h} R(\phi) u \mathrm{~d} h
$$

where $\boldsymbol{R}(\phi)$ is the specific resistance of the elemental layer $\mathrm{d} h$ and $h_{0}$ is the cake height at $t=0$. Differentiating both sides of eq. (7) with respect to time and substituting for $\mathrm{d} h$ from eq. (5) we obtain

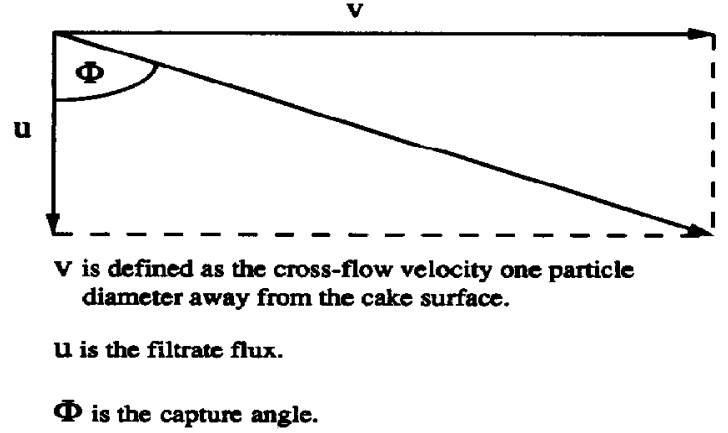

Fig. 10. Definition of the capture angle.

$$
-\frac{\Delta P \mathrm{~d} u}{\lambda u^{3} f(\phi) R(\phi)}=\mathrm{d} t
$$

Equations (5) and (8) now provide a mathematical description of cake height and filtrate flux kinetics.

\section{Non-dimensional presentation}

The numerical and experimental data can be normalised to initial conditions at an arbitrary time $t=t_{0}$, such that the governing equations can be written in terms of the dimensionless parameters

$$
\begin{array}{cl}
u^{*}=\frac{u}{u_{\mathrm{o}}}, & h^{*}=\frac{h}{h_{\mathrm{o}}}, \quad t^{*}=\frac{\left(t-t_{\mathrm{o}}\right) u_{0}}{h_{\mathrm{o}}} \\
v^{*}=\frac{v}{u_{\mathrm{o}}}, & R^{*}=\frac{R}{R_{\mathrm{o}}} \quad \text { and } \quad R^{*}(\phi)=\frac{R(\phi)}{R_{\mathrm{o}}}
\end{array}
$$

where $u_{0}$ and $h_{0}$ are the flux and cake height at the arbitrary time $t=t_{0}$ and $R_{0}=\Delta P /\left(u_{0} h_{0}\right)$. Hence, $u^{*}=h^{*}=1$ at $t^{*}=0$.

Equations (5) and (8), respectively, can be now written in non-dimensional form as

$$
\mathrm{d} h^{*}=\lambda u^{*} f(\phi) \mathrm{d} t^{*}
$$

and

$$
-\frac{\mathrm{d} u^{*}}{\lambda u^{* 3} f(\phi) R^{*}(\phi)}=\mathrm{d} t^{*} .
$$

The value of the constant $\lambda$ can be simply determined from data obtained under conditions of no applied cross-flow as $f(\phi)$ and $R^{*}(\phi) \rightarrow 1$ giving

$$
u^{*}=\left(2 \lambda t^{*}+1\right)^{-1 / 2} \text {. }
$$

The dimensionless time origin was fixed at the experimental time of $60 \mathrm{~s}$ as this was the earliest time at which reliable values of both $u$ and $h$ could be obtained. Having chosen a suitable time at which to set $u_{0}$ and $h_{0}$, the filtrate flux data for $v=0$ in Fig. 6 was normalised to give $u^{*}$ vs $t^{*}$. Using a least-squares fitting routine with $\lambda$ as a variable the data was fitted to the form of eq. (11) and a best-fit value of $\lambda=4.2$ $\times 10^{-4}$ was obtained. Given that, at early times, the uncertainty in $h$ is high (i.e. $\sim \pm 20 \%$ ) this $\lambda$ value is in reasonable agreement with that obtained from the 
raw data and so it was subsequently adhered to in the modelling. As $\lambda=s /(1-\varepsilon-s)$, keeping it fixed implies that the void fraction $(\varepsilon)$ is constant which is not strictly true in the case when the resistance $(R)$ varies. However, it is assumed that the effect of $\varepsilon$ will dominate in the resistance as, for example, the CarmanKozeny relationship gives $R \propto\left(1-\varepsilon^{2}\right) / \varepsilon^{3}$ (Carman, 1956) and that in $\lambda$ the voidage can be represented by a mean value $\bar{\varepsilon}$.

Thus, if $f(\phi)$ and $R^{*}(\phi)$ can be expressed as functions of $u^{*}$, eq. (10) can be integrated to obtain the relationship between dimensionless flux and time, which can in turn be used to calculate $h^{*}$ in eq. (9). This process has been performed numerically so that the sensitivity to the functional forms $f(\phi)$ and $R^{*}(\phi)$ could be examined.

\section{Selection of $\mathrm{f}(\phi)$ and $\mathrm{R}^{*}(\phi)$}

The capture probability function. The form of $f(\phi)$ will determine the predicted growth of $h^{*}$. For example, when all particles are captured $f(\phi)=1$ and eq. (9) collapses to the static filtration case, whereas if few particles are captured $f(\phi) \Rightarrow 0$ and cake height growth ceases. For our system, $f(\phi)=1$ corresponds to the early time behaviour when the filtrate flux $(u)$ is dominant, and $f(\phi) \Rightarrow 0$ represents the approach to limiting conditions where the cross-flow velocity $(v)$ dominates. Between these two extremes, our observations suggest that the capture probability is determined by the relative magnitudes of $u$ and $v$. To describe this behaviour, a suitable although somewhat arbitrary function for the capture probability was tried,

$$
f(\phi)=\cos (\phi)=\frac{u}{\left(u^{2}+v^{2}\right)^{1 / 2}}=\frac{u^{*}}{\left(u^{* 2}+v^{* 2}\right)^{1 / 2}}
$$

In the first instance we may isolate the effect of $f(\phi)$ by assuming a constant specific cake resistance, i.e. putting $R^{*}(\phi)=R^{*}=1$ in eq. (10). In this case the simple Darcy law is applicable, which in dimensionless form becomes

$$
h^{*}=\frac{1}{u^{*}}
$$

Under these conditions, the capture probability function given in eq. (11) has been used to predict $u^{*}$, and hence $h^{*}$ as function of time, and the results are shown in Fig. 11(a) and (b), respectively. The values of dimensionless velocity $v^{*}$ represent the same constant crossflow conditions as those used experimentally to obtain the data in Figs 5 and 6. It can be seen that inclusion of the $\cos (\phi)$ capture factor in the solid mass balance leads to the prediction of reduced cake height with increasing cross-flow, and as the specific cake resistance is constant a corresponding increase in filtrate flux is obtained.

Another form of the capture factor can be proposed by referring more closely to the evidence of the optical observations. It was shown that particle rolling only took place at later times when the cross-flow dominated the fluid mechanics. By watching the cake formation videos in slow motion, still frames were carefully selected at the times where particle rolling was first noticed. The angle between the impacting particle trajectories and the cake surface was then measured and $\phi$ was found to be $\sim 86^{\circ} \pm 2^{\circ}$. Hence, a more realistic capture probability function would be

$$
f(\phi)= \begin{cases}1 & \text { for } 0^{\circ} \leqslant \phi<\phi_{c} \\ \cos (\alpha) & \text { for } \phi_{c} \leqslant \phi \leqslant 90^{\circ}\end{cases}
$$

where

$$
\alpha=\frac{90^{\circ}\left(\phi-\phi_{c}\right)}{\left(90^{\circ}-\phi_{c}\right)}
$$

where $\phi_{c}$ is the critical angle at which particle rolling commences, in this case $86^{\circ}$. Here, all impacting particles are captured until a critical approach angle is reached, then the capture probability diminishes reaching 0 when $\phi=90^{\circ}$. This form of $f(\phi)$ produces the flux and cake height kinetics shown in Fig. 12(a)
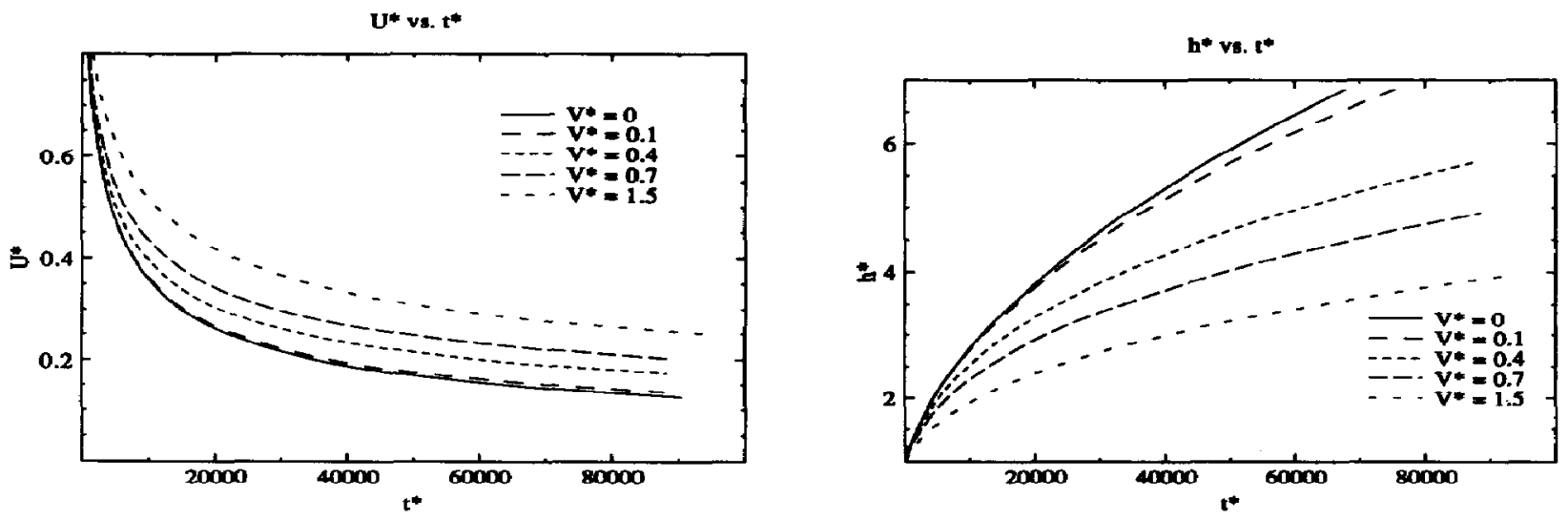

Fig. 11. (a) $U^{*}$ vs $t^{*}$. (b) $h^{*}$ vs $t^{*}$. $f(\phi)=\cos (\phi), R^{*}(\phi)=1$ and $\lambda=4.2 \times 10^{-4}$. 
and (b), respectively. As found for the previous function, when the cross-flow velocity is increased, the filtrate flux increases and the cake height decreases. However, when compared with Fig. 11(a) and (b) it can be seen that the effect is more subtle and limited to the later times of the filtration process. Other forms of $f(\phi)$ were examined including the use of $\cos ^{2}(\phi)$ and $\cos ^{2}(\alpha)$ in eqs (12) and (14) and the general trends of $u^{*}$ and $h^{*}$ were unchanged except the that effects of $v^{*}$ were slightly more marked at lower $t^{*}$ values compared with Figs 11(a) and (b) and 12(a) and (b).

Thus, the capture probability function alone does not produce predictions consistent with our experimental results as, although the cake height is reduced by increasing the cross-flow velocity, the corresponding filtrate flux increases. However, the time scale and extent of this effect can be controlled by the form of $f(\phi)$ chosen and that given by eqs (14a) and (14b) is plausible from our experimental evidence.

The specific resistance function. The form of $R(\phi)$ must reflect the manner in which impact trajectory might affect particle packing. It was seen that in the early stages of filtration, cake formation occurred in a non-selective packing manner but as the cross-flow velocity became more significant at the cake-suspension interface, a more selective, and hence more efficient packing process took place. Further the hydraulic resistance ratio for a dynamically grown to a statically formed cake was found to be $\sim(3 \pm 0.5)$. Thus, we would expect $R$ to be equivalent to the static cake value when $\phi=0^{\circ}$ and increase with capture angle up to a limiting dynamic value as $\phi \Rightarrow 90^{\circ}$. A suitable function is

$$
R^{*}(\phi)=R^{\prime}-\left(R^{\prime}-1\right) \cos (\phi)
$$

where $R^{*}(\phi)=R(\phi) / R_{1}, R^{\prime}=R_{2} / R_{1}$ and $R_{2}$ is the resistance of cake layers formed at high cross-flow with negligible filtrate flux and $R_{1}$ is the resistance of cake formed with no applied cross-flow. This assumes that $R$ at $t=0$ is the zero cross-flow value, i.e. $R_{0} \sim R_{1}$, which is valid provided that the time origin is fixed early in the filtration process when particle

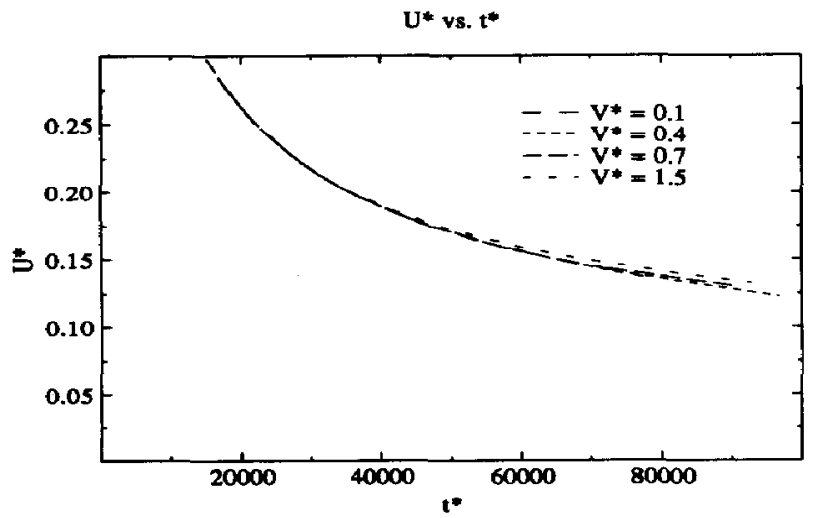

packing is still non-selective. Thus if $\phi=0^{\circ}$ eq. (15) collapses to the static case $R^{*}(\phi)=1$ and as $\phi \Rightarrow 90^{\circ} R^{*}(\phi) \Rightarrow R^{\prime}$ which has been evaluated experimentally. The effect of $R^{*}(\phi)$ in eqs $(9)$ and $(10)$ was isolated by putting $f(\phi)=1$, i.e. all particles captured, and using $R^{\prime}=3$ in eq. (15) the predictions of $u^{*}$ and $h^{*}$ kinetics shown in Fig. 13(a) and (b) were obtained. In this case both the filtrate flux and cake height decrease with increasing cross-flow velocity, in agreement with the trends found for the experimental data.

Similarly to $f(\phi)$, other resistance functions were explored using the forms $R^{*}(\phi)=R^{\prime}-\left(R^{\prime}-1\right)$ $\cos ^{2}(\phi)$, and a linear trend with angle, i.e. $R^{*}(\phi)=1$ $+2 \phi / \pi\left(R^{\prime}-1\right)$. The first case slightly increased the rapidity of the initial $u^{*}$ decline with corresponding decrease in $h^{*}$ growth, and broadened the spread of the flux and height curves with $v^{*}$ in the $t^{*} \sim(1$ to 3$)$ $\times 10^{4}$ region. When the linear trend was applied the initial flux decline was much steeper than that shown in Fig. 13(a) and spreading of the curves with $v^{*}$ was most noticable in the range $t^{*} \sim 0-2 \times 10^{4}$. Otherwise the general prediction of decreasing flux and cake height with increasing cross-flow velocity was not changed. As found for $f(\phi)$ then, the form of $R^{*}(\phi)$ simply controls the time scale and extent of the resistance effect.

Combination of $\mathrm{f}(\phi)$ and $\mathrm{R}^{*}(\phi)$. The combined effect of the surface capture factor expressed by eq. (14) and the change in specific resistance expressed by eq. (15) is shown in Fig. 14(a) and (b). In this situation, the model is compared with the experimental data. Having chosen the form of the functions $f(\phi)$ given by eqs (14) and (15), respectively, the only remaining adjustable parameter is the ratio of the specific resistances $R^{\prime}=R_{2} / R_{1}$. A trial-and-crror fitting procedure was applied to the model and data within a range $R^{\prime}=2-3.75$ in increments of 0.25 . The best overall fit was obtained for $R^{\prime}=3.25$ and this value has been used for the model data shown in Fig. 14(a) and (b). Agreement between model and data is considered to be good. The experimental error in the flux data is commensurate with the size of the symbols used and it

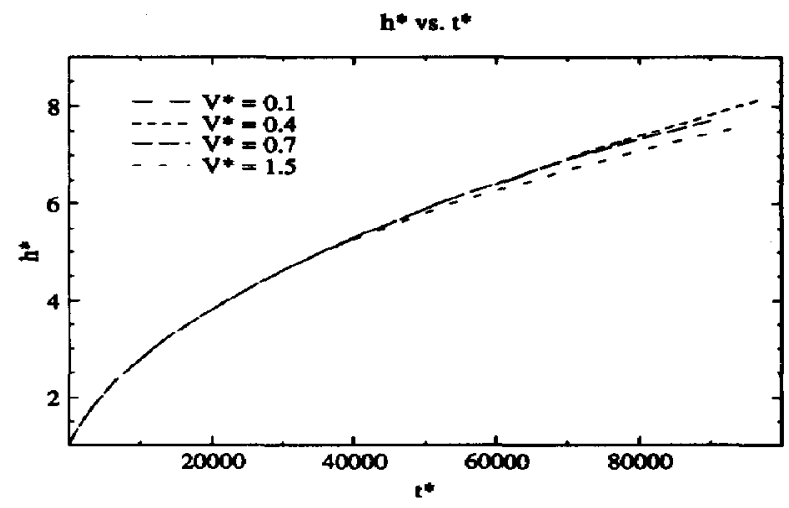

Fig. 12. (a) $U^{*}$ vs $t^{*}$. (b) $h^{*}$ vs $t^{*}$. $f(\phi)=1\left\{\right.$ for $\left.0^{\circ} \leqslant \phi<86^{\circ}\right\}, f(\phi)=\cos (\alpha)\left\{\right.$ for $\left.86^{\circ} \leqslant \phi \leqslant 90^{\circ}\right\}, R^{*}(\phi)$ $=1$ and $\lambda=4.2 \times 10^{-4}$. 

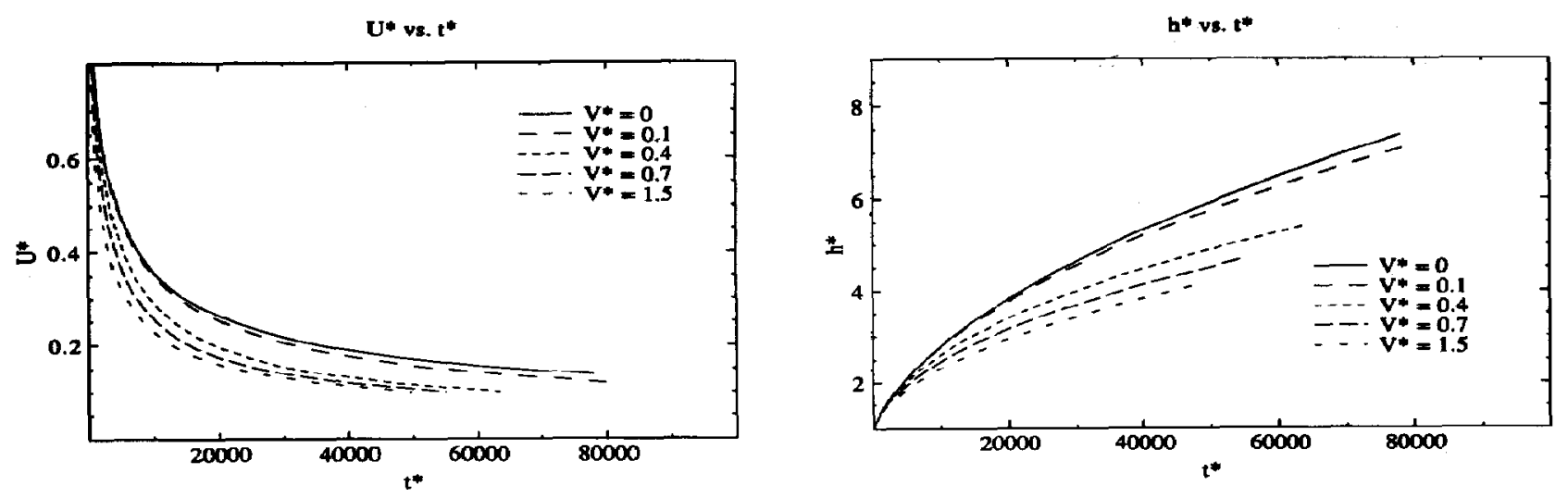

Fig. 13. (a) $U^{*}$ vs $t^{*}$. (b) $h^{*}$ vs $t^{*}, f(\phi)=1, R^{*}(\phi)=R^{\prime}-\left(R^{\prime}-1\right) \cos (\phi), R^{\prime}=3$, and $\lambda=4.2 \times 10^{-4}$.
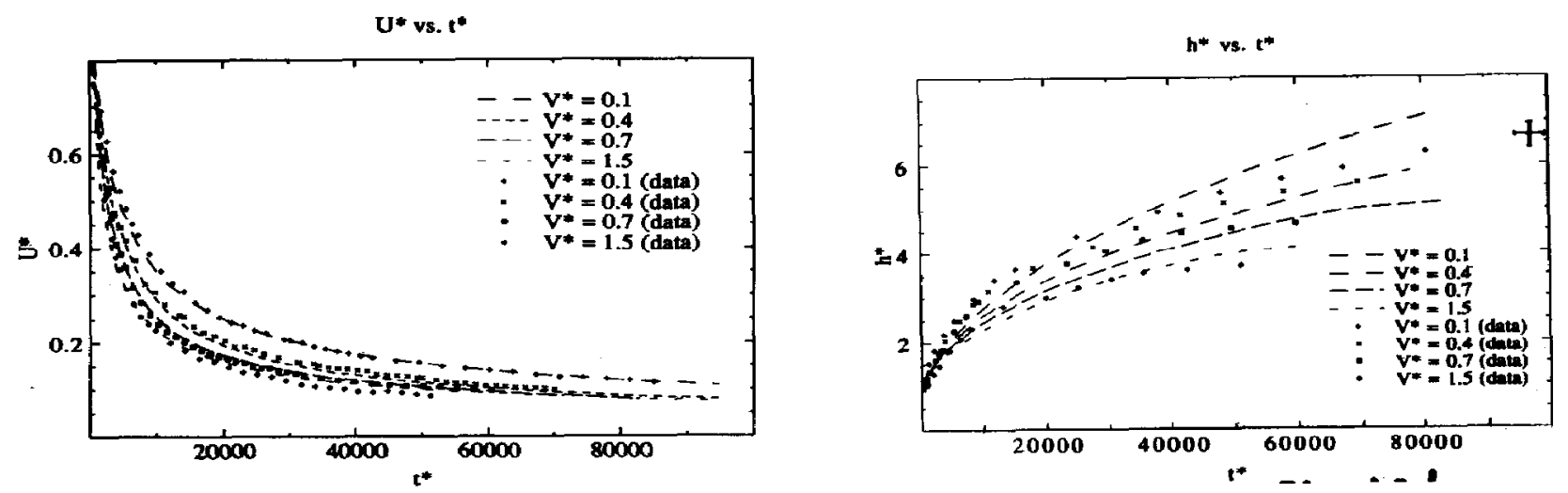

Fig. 14. (a) $U^{*}$ vs $t^{*}$. (b) $h^{*}$ vs $t^{*}$. Comparison of data with model. $f(\phi)=1$ for $\left.0^{\circ} \leqslant \phi<86^{\circ}\right\}$, $f(\phi)=\cos (\alpha)\left\{\right.$ for $\left.86^{\circ} \leqslant \phi \leqslant 90^{\circ}\right\}, R^{*}(\phi)=R^{\prime}-\left(R^{\prime}-1\right) \cos (\phi), R^{\prime}=3.25$, and $\lambda=4.2 \times 10^{-4}$.

can be seen that the model is in good agreement with the flux data. In addition, the value of $R^{\prime}=3.25$ is in close agreement with the independently experimentally measured ratio $R^{\prime}=(3 \pm 0.5)$ reported in this paper. For large $t^{*}$, however, there is a noticeable over prediction of $u^{*}$ for $v^{*}=1.5$. It is possible that particles on the cake surface may also under go further re-arrangement in the longer term under the influence of fluid shear. The relative magnitude of this effect is then likely to be more marked for higher cross-flow velocities where cake growth has virtually ceased.

The predicted model cake heights shown in Fig. 14(b) show qualitative agreement with the reported data and generally fall within the experimental error of the measurements. For the particular model system we have examined here the cake resistance term $R^{*}(\phi)$ is more dominant than the surface capture factor $f(\phi)$.

\section{DISCUSSION AND CONCLUSIONS}

We have shown that, for the dilute suspension of polyethylene particles examined, cross-flow filtration kinetics appear to be controlled by two factors, namely, the capture behaviour at the fluid-solid interface and the manner in which the particles pack when captured at the cake surface. Both terms depend on complicated interfacial parameters which are unlikely to be easily predicted. We have taken a pragmatic view and modelled the behaviour around our experimental observations. In order to describe the global results, it has been necessary to develop two functions, the capture factor $f(\phi)$ and the specific resistance function $R^{*}(\phi)$. These two terms are a direct consequence of our experimental observations summarised below.

(i) Particles were drawn towards the filter cake in a manner determined by the relative magnitudes of the cross-flow velocity and the filtrate flux.

(ii) When the flux dominated, particles were advected to the cake surface and attached at the impact site. In this regime packing was non-selective.

(iii) In the dynamic regime, when the cross-flow dominated, particles were seen to roll along the cake surface until captured at a stable site and eventually a regime was reached where particles impacting upon 
the cake did not necessarily attach to it. In this case the packing appeared highly selective.

(iv) No evidence of particle back diffusion was seen.

The fact that the specific cake resistance of a cake formed under cross-fiow was found to be three times greater than that formed under static conditions is a clear evidence that cross-flow can influence the cake particle packing. The direct optical observations show how this can come about in that at high cross-flow the particles are highly selective as to which site they will choose to transform from the fluid to the cake, whereas when no cross-flow is present the particles have little or no scope for packing adjustment. This selectivity during cross-flow will depend on a wide range of factors involving particle size and form, surface topology and particle interfacial forces.

Assuming that the resistance effect is caused by a change in packing arrangement, it is useful to assess the credibility of the measured ratio $R^{\prime}$. We might expect the zero and high cross-flow cake voidage fractions ( $\varepsilon$ ) to lie in a range bounded by the random loose packing (RLP) limit and some highly ordered limit, e.g. hexagonal close packing (HCP), although the latter is only strictly applicable for spherical particles. The RLP limit for $\varepsilon$ has recently been determined for a neutrally buoyant suspension by Onoda and Liniger (1990) as being $\sim 0.44$; the equivalent figure for HCP is 0.26. Assuming that the cake resistance can be expressed by the Carman-Kozeny equation, we would expect $R \propto(1-\varepsilon)^{2} / \varepsilon^{3}$ and using the above $\varepsilon$ values this predicts $\boldsymbol{R}_{\mathrm{HCP}} / \boldsymbol{R}_{\mathrm{RLP}} \sim 8.5$. This represents the maximum ratio and as our experimental figure $R^{\prime} \sim(3 \pm 0.5)$ is below this, it is credible because we would not expect the static or dynamic conditions to produce cakes of perfect loose or close packing.

The fact that no particle back flux was observed is also very significant as it indicates that our system cannot be described in terms of a diffusion balance mechanism. This view is further supported by the result that the dynamic filtrate flux scaled with filter length $L$, in contrast to the $L^{2 / 3}$ dependence that would be expected for a system controlled by concentration polarisation [e.g. see Davis and Birdsell (1987) or Pearson and Sherwood (1988)]. The actual mechanism observed in (iii) above for the dynamic regime is more closely related to the particle adhesion description of cake formation, for example the critical flow model of Rautenbach and Schock (1988) or the force balance model of Blake et al. (1990). However, in these models an abrupt transition between adhesion to and rolling across the cake exists at some critical value of the cross-flow velocity or net tangential force. This is not the case for our observations. Although it was possible to detect a critical condition for the onset of particle rolling, the transition from the situation where all impacting particles attached to the cake to one where the majority did not was a smooth one, best described as a decreasing probability of adhesion or capture. Ideally a sharp transition would exist if conditions were perfectly uniform along the whole cake, but particle adhesion at any individual site would be influenced by local surface topology, which may vary with packing arrangement and particle-size polydispersity, and the consequent particle interactions. This means that any global description of the cake growth would have to cover a spectrum of critical conditions. The concept of decreasing particle capture probability provides just such a description and would therefore appear to provide a sound basis for modelling of our system, along with the selective packing mechanism. This is borne out by the generally good agreement with the experimental data shown in Fig. 14(a) and (b).

For the model suspension tested we have shown that if plausible forms of capture probability and resistance function can be found, based on observations of the relevant cake formation mechanisms, the mathematical model presented here can provide a good description of the observed experimental behaviour. Further, this model may be applicable to other systems if suitable adjustment is made to the forms of $f(\phi)$ and $R^{*}(\phi)$. An example can be seen in the earlier assessment of $f(\phi)$ as when $R^{*}(\phi) \rightarrow 1$, i.e. a very weak resistance effect, the more usual result of increasing filtrate flux with increasing cross-flow velocity was predicted. When this is so, the evaluation of the critical angle at which particle capture probability starts to fall from unity may be sufficient to describe the system. However, the limit of $f(\phi) \rightarrow 0$ as $\phi \rightarrow 90^{\circ}$ may also need examination as it may be that particle capture may cease before $\phi=90^{\circ}$ as for example from the results obtained with polystyrene latices by Blake et al. (1990) for dynamic flux as a function of wall shear stress, it is possible to calculate the limiting angle to be in the range $\phi_{c} \sim 87.9^{\circ}-89.9^{\circ}$. This limiting angle would need to be included in the form of the capture factor in order to predict a steady-state flux in the limit of infinite time.

Particle size should also be a relevant factor in the choice of suitable capture and resistance functions. In our model, the capture angle is sensitive to particle size because $v$ is defined as the cross-flow velocity at one particle diameter away from the cake surface. This indicates that under identical $u$ and $v$ conditions, small particles could form slightly thicker cakes than larger ones. However, as smaller particles produce cakes of higher resistance, a given mean applied crossflow velocity does not give rise to similar fluxes in both the small and large particle cases and we might expect the effect of the intrinsic cake resistance to be dominant over any small effect caused by the arbitrary definition of $v$. Particle size should also effect the resistance function dependence on cross-flow. Wakeman and Tarleton (1991) found that for calcite of mean particle size $27.5 \mu \mathrm{m}$, an increase in cross-flow velocity led to a decrease in filtrate flux whereas for a mean size of $2.7 \mu \mathrm{m}$, the opposite was true. This indicates that the effect of packing selectivity is more marked for the larger size compared to the smaller. An explanation can be found in that the smaller the 
particle, the larger the number of suitable packing sites per unit length of filter. Thus, for a given crossflow velocity the smaller particle will sample more sites per unit time and have a higher probability of capture. Also Brownian motion will become more significant for smaller particles, perhaps allowing more sites to be explored by them. Hence, efficient packing can be achieved at the lower cross-flow velocities, so any increase in $v$ simply reduces the dynamic cake height and cannot change its porosity profile. Thus, in order to apply our mathematical model, in principle the ratio $R^{\prime}$ would have to be evaluated for different particle sizes.

Acknowledgements-The authors wish to acknowledge the SERC and Schlumberger Cambridge Research (SCR) for funding the project. We would particularly like to thank Dr. G. Maitland, Dr. G. Meeten and the Rock and Fluid Physics Group at SCR for their collaboration and finally, we wish to thank Ted Roberts (Department of Chemical Engineering Cambridge) for many useful discussions on the modelling work.

\section{NOTATION}

$d \quad$ particle diameter

$f(\phi)$ capture probability function

$h$ cake height

$H$ channel height

$M \quad$ cumulative filtrate mass

$\Delta P$ pressure drop across cake

$R \quad$ specific cake resistance

$R_{1} \quad$ zero cross-flow resistance

$R_{2}$ high cross-flow resistance

$R^{\prime} \quad R_{2} / R_{1}$

$R(\phi) \quad$ resistance function

$R e \quad$ Reynolds number

$s \quad$ solid volume fraction

$t$ time

$u$ filtrate flux

$v \quad$ cross-flow velocity at one particle diameter from the cake surface

$\bar{v} \quad$ mean channel cross-flow velocity

\section{Greek letters}

$\gamma \quad$ shear rate

$\varepsilon \quad$ cake voidage fraction

$\bar{\varepsilon} \quad$ mean cake voidage

$\eta \quad$ fluid viscosity

$\lambda \quad=s /(1-\varepsilon-s)$

$\rho$ fluid density

$\phi \quad$ capture angle

$\phi_{c} \quad$ critical angle for the start of particle rolling

\section{Sub and superscripts}

$0 \quad$ value at $t=0$

* parameter made dimensionless using $t=0$ value

\section{REFERENCES}

Akers, R. J. and Ward, A. S., 1977. Liquid filtration theory and filtration pretreatment, in Filtration Principles and
Practices (Edited by Clyde Orr), Part 1, pp. 169-186, Marcel Dekker, New York.

Bagdasarian, A., Tiller, F. M. and Donavan, J., 1977. High pressure, thin cake, staged filtration. Filtr. Sep. 14(5), 203-206.

Blake, N. J., Cumming. I. W. and Streat, M., 1990. Prediction of, steady state cross-flow filtration using a force balance model, in Proceedings of 5 th World Filtration Congress, Nice.

Carman, P. C., 1956. Flow of Gases Through Porous Media. Academic Press, New York.

Colton, C. K., Henderson, L. W., Ford, C. A. and Lysaght, M. J., 1975. Kinetics of hemodiafiltration. 1. In vitro transport characteristics of a hollow fibre blood ultrafilter. J. Lab. Clin. Med. 85, 355.

Davis, R. H. and Birdsell, S. A., 1987. Hydrodynamic model and experiments for cross-flow microfiltration. Chem. Engng Cormmun. 49, 217-234.

Davis, R. H. and Sherwood, J. D., 1990. A similarity solution for steady state cross-flow microfiltration. Chem. Engng Sci. 45(11), 3203-3209.

Eckstein, E. C., Bailey, D. G. and Shapiro, A. N., 1977. Self diffusion of particles in shear flow of a suspension. $J$. Fluid Mech. 79, 191-208.

Fane, A. G., 1984. Ultrafiltration of suspensions. J. Membrane Sci. 20, 249-259.

Fischer, E. and Raasch, J., 1985. Cross-flow filtration. Ger. chem. Engng 8, 211-216.

Fordham, E. J. and Ladva, H. K. J., 1989. Cross-flow filtration of bentonite suspensions. PhysicoChem. Hydrodyn. 11(4), 411-439.

Gray, G. R. and Darley, H. C. H., 1980. Composition and Properties of Oil Well Drilling Fluids, 4th Edition. Gulf, Houston, TX.

Green, G. and Belfort, G., 1980. Fouling of ultrafiltration membranes; lateral migration and the particle trajectory model. Desalination 35, 129-147.

Gutman, R. G., 1977. The design of membrane separation plant. Chem. Engr. 322, 510-513 and 521-523.

Hoogland, M. R., Fell, C. J. D., Fane, A. G. and Jones, D. A. R., 1990. The optimum design of cross-flow filtration elements for mineral slurry processing, in Proceedings of 5th World Filtration Congress, Nice. pp. 604-610.

Hunt, J. W., Brouckaert, C. J., Raal, J. D. and Buckley, C. A., 1987. The unsteady-state modelling of cross-flow microfiltration. Desalination 64, 431-442.

Lu, W-M. and Ju, S-C., 1989. Selective particle deposition in crossflow filtration. Sep. Sci. Technol. 24(7/8), 517-540.

Nakao, S., Nomura, S. and Kimura, S., 1990. Transport phenomena of the cross-flow microfiltration process, in Proceedings of 5th World Filtration Congress, Nice, pp. $564-570$.

Onoda, G. Y. and Liniger, E. G., 1990. Random loose packings of uniform spheres and the dilatancy onset. Phys. Rev. Lett. 64(22), 2727-2730.

Pearson, J. R. A. and Sherwood, J. D., 1988. Continuum modelling of cross-flow filtration. PhysicoChem. Hydrodyn. 10(5/6), 647-661.

Philip, J. R. and Smiles, D. E., 1982. Macroscopic analysis of the behaviour of colloidal suspensions. Adv. Colloid Interface Sci. 17, 83-103.

Porter, M. C., 1972a. Ultrafiltration of colloidal suspensions. A.I.Ch.E. Symp. Ser. 68, 21-30.

Porter, M. C., 1972b. Concentration polarisation with membrane ultrafiltration. Ind. Engng Chem. Prod. Res. Dev. 11, 234-248.

Porter, M. C. and Nelson, L., 1972. Ultrafiltration in the chemical, food processing, pharmaceutical and medical industries, in Recent Developments in Separation Science, Vol. 2, p. 227. CRC Press, Cleveland, OH

Rautenbach, $R$. and Schock, G., 1988. Ultrafiltration of macromolecular solutions and cross-flow microfiltration of colloidal suspensions: a contribution to permeate flux calculations. J. Membrane Sci. 36, 231-242. 
Romero, C. A. and Davis, R. H., 1988. Global model of crossflow microfiltration based on hydrodynamic particle diffusion. J. Membrane Sci. 39, 157-185.

Romero, C. A. and Davis, R. H. 1990. Transient model of cross-flow microfiltration. Chem. Engng Sci. 45, 13-25.

Schmitz, P., Gouveneur, C., Houi D. and Madianos M., 1990. Theoretical model at pore scale for particle deposition on a cross-flow filtration membrane, in Proceedings of 5th World Filtration Congress, Nice, pp. 571-578.

Tanny, G. B., Hauk, D. and Meuin, U., 1982. Biotechnical applications of a pleated cross-flow microfiltration module. Desalination 41, 299-312.
Treffry-Goatly, K., Buchan, M. I., Renchen, G. E. and Buckley, C. A., 1987. The dewatering of sludges using a tubular filter press. Desalination 67, 467-479.

Wakeman, R. J. and Tarleton, E. S., 1991. Colloidal fouling of microfiltration membranes during the treatment of aqueous feed streams. Desalination (submitted).

Zydney, A. L. and Colton, C. K., 1986. A concentration polarisation model for the filtrate flux in cross-flow microfiltration of particulate suspensions. Chem. Engng Commun. 47, 1-21. 